\title{
Neurotoxic effects of ecstasy on the thalamus
}

Maartje M. L. de Win, Gerry Jager, Jan Booij, Liesbeth Reneman, Thelma Schilt, Cristina Lavini, Sílvia D. Olabarriaga, Nick F. Ramsey, Gerard J. den Heeten and Wim van den Brink

\section{Background}

Neurotoxic effects of ecstasy have been reported, although it remains unclear whether effects can be attributed to ecstasy, other recreational drugs or a combination of these.

\begin{abstract}
Aims
To assess specific/independent neurotoxic effects of heavy ecstasy use and contributions of amphetamine, cocaine and cannabis as part of The Netherlands XTC Toxicity (NeXT) study.

\section{Method}

Effects of ecstasy and other substances were assessed with ${ }^{1} \mathrm{H}$-magnetic resonance spectroscopy, diffusion tensor imaging, perfusion weighted imaging and $\left[{ }^{123} \mid\right] 2 \beta$ -

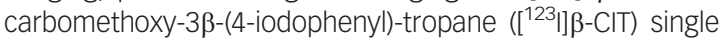
photon emission computed tomography (serotonin
\end{abstract}

transporters) in a sample $(n=71)$ with broad variation in drug use, using multiple regression analyses.

\section{Results}

Ecstasy showed specific effects in the thalamus with decreased $\left[{ }^{123} \mid\right] \beta$-CIT binding, suggesting serotonergic axonal damage; decreased fractional anisotropy, suggesting axonal loss; and increased cerebral blood volume probably caused by serotonin depletion. Ecstasy had no effect on brain metabolites and apparent diffusion coefficients.

\section{Conclusions}

Converging evidence was found for a specific toxic effect of ecstasy on serotonergic axons in the thalamus.

\section{Declaration of interest}

None. Funding detailed in Acknowledgements.
Previous studies have suggested (serotonergic) neurotoxicity of the recreational drug ecstasy (3,4-methylenedioxymethamphetamine, MDMA). ${ }^{1,2}$ However, given that most ecstasy users are polydrug users, these findings are still debated as few have adequately controlled for this. ${ }^{3}$ The current study, part of The Netherlands XTC Toxicity (NeXT) study, ${ }^{4}$ was designed to control for polydrug use by including a sample that varied in the type and amount of drugs used. The relatively low correlations between the use of ecstasy and other substances allowed for the use of linear multiple regression analysis to differentiate between the effects of ecstasy and of other substances without problems of multicollinearity. A combination of both single photon emission computed tomography (SPECT) and advanced magnetic resonance imaging (MRI) techniques was used to simultaneously assess structural and functional aspects of potential ecstasy-induced neurotoxicity.

\section{Method}

\section{Participants}

In total, 71 participants (44 male, 27 female; mean age 23 years, s.d. $=3.8$, range 18-37) were included. A detailed description of the recruitment procedure can be found in a special design paper on the NeXT study. ${ }^{4}$

Recruitment aimed to include a sample of individuals with variations in the amount and type of drugs used to keep correlations between the different drugs as low as possible. Potential candidates interested in participating in the study were asked to fill out a questionnaire on their drug use, but were masked to the inclusion criteria. Besides the typical heavy polysubstance ecstasy users, preference was given to candidates who were either 'selective ecstasy users' (100 ecstasy pills or more lifetime, but no or almost no use of other drugs except for cannabis) or 'polydrug-but-no-ecstasy users' (extensive experience with amphetamine and/or cocaine, but (almost) no ecstasy use, i.e. $<10$ pills lifetime). In the end, the sample included 33 heavy ecstasy users and 38 non-ecstasy users, with both ecstasy users and non-ecstasy users showing considerable variation in type and amount of other drugs taken, for example some heavy ecstasy users reported minimal use of other drugs such as cannabis, amphetamine or cocaine, whereas others were moderate or frequent users of one or more other psychoactive drugs. Similarly, some ecstasy-naïve individuals used no drugs at all, whereas other ecstasy-naïve individuals reported incidental or frequent use of amphetamine and/or cocaine and/or cannabis. Individuals were recruited using a combination of targeted site sampling, advertisement and snowball sampling. All participants had to be between 18 and 35 years of age. Exclusion criteria included severe medical or neuropsychiatric disorders; use of psychotropic medications affecting the serotonin system; pregnancy; use of intravenous drugs; and contraindications for MRI. Participants had to abstain from using psychoactive substances for at least 2 weeks and from alcohol for at least 1 week before examinations. Pre-study abstinence was checked with urine drug screening (enzyme-multiplied immunoassay for amphetamines, MDMA, opioids, cocaine, benzodiazepines, cannabis and alcohol).

Besides SPECT and MRI, individuals underwent functional MRI and cognitive testing reported in separate publications. ${ }^{5,6}$ Participants were paid to participate ( $€ 150$ for 2 days). The study was approved by the local medical ethics committee and written informed consent from each person was obtained according to the Declaration of Helsinki.

\section{Assessment of ecstasy use and potential confounders}

Lifetime use of ecstasy (number of tablets), cannabis (number of 'joints'), amphetamines (number of occasions), cocaine (number of occasions), and use of alcohol (units/week) and tobacco (cigarettes/week) were assessed using substance-use questionnaires and the Substance Abuse Scales of the Mini International Neuropsychiatric Interview (MINI, version 5$)^{7}$ for DSM-IV clinical disorders. Hair samples were collected from all but 19 participants (hair too short or hair dyed) for analysis on previous ecstasy 
use (gas chromatography/mass spectroscopy). Hair analyses $(n=52)$ confirmed previous ecstasy use in $86 \%$ of individuals who reported to have used ecstasy. In addition, results from hair analyses showed no evidence for previous use of ecstasy in $96 \%$ of participants who had reported being ecstasy-naïve. Altogether, agreement between self-reported ecstasy use and ecstasy use according to hair analyses was $90 \%$, resulting in a kappa of 0.81 , which represents excellent overall chance adjusted agreement. Verbal IQ was estimated using the Dutch version of the National Adult Reading Test. ${ }^{8}$

\section{MRI acquisition and post-processing}

\section{Acquisition}

Magnetic resonance imaging was performed on a $1.5 \mathrm{~T}$ scanner (Signa Horizon, LX 9.0, General Electric Medical Systems, Milwaukee, Wisconsin) using the standard head coil. Acquisition, post-processing and quality control were performed with the same methods as used in another substudy of the NeXT study. ${ }^{9}$ For completeness, we have summarised the most relevant aspects of the employed methods. The protocol included axial proton density- and $\mathrm{T}_{2}$-weigthed imaging; three voxel-based proton magnetic resonance spectroscopy ( $\left.{ }^{1} \mathrm{H}-\mathrm{MRS}\right)$ scans; diffusion tensor imaging; perfusion-weighted imaging; and high-resolution $\mathrm{T}_{1}$-weigthed $3 \mathrm{D}$ imaging. The ${ }^{1} \mathrm{H}$-MRS voxels were placed in the left centrum semiovale (frontoparietal white matter) and in mid-frontal and mid-occipital grey matter in analogy to previous studies. ${ }^{10,11}$ Throughout the study, positioning of participants in the scanner and positioning of the slices and voxels were performed by the same examiner and according to protocol in order to keep positioning as reproducible as possible.

\section{Post-processing}

Spectra derived from ${ }^{1} \mathrm{H}-\mathrm{MRS}$ were analysed using Linear Combination of Model spectra (LCModel). ${ }^{12}$ Ratios of N-acetylaspartate (NAA; neuronal marker), choline (Cho; reflecting cellular density) and myoinositol ( $\mathrm{mI}$; marker for gliosis) relative to (phospho)creatine $(\mathrm{Cr})$ were calculated.

Apparent diffusion coefficient and fractional anisotropy maps were calculated from the diffusion tensor imaging ${ }^{13}$ and cerebral blood volume maps from the perfusion-weighted imaging scans. Fractional anisotropy, apparent diffusion coefficient and cerebral blood volume were spatially normalised by registration to the Montreal Neurological Institute brain template (MNI152), and segmentation was performed to separate into cerebral spinal fluid, white and grey matter (Fig. 1). The cerebral blood volume maps were intensity-scaled to mean individual cerebral blood volume intensity of white matter derived from the segmentation procedure to generate relative cerebral blood volume maps.

Regions of interest (ROIs) were drawn on the MNI152 brain template in thalamus, putamen, globus pallidus, head of the caudate nucleus, centrum semiovale (frontoparietal white matter), and dorsolateral frontal, mid-frontal, occipital, superior parietal, and temporal cortex (Fig. 2). Only grey matter voxels were included for the cortical ROIs, whereas white and grey matter voxels were included for the ROIs of the basal ganglia (i.e. excluding cerebral spinal fluid voxels). Selection of ROIs was based on findings of previous studies, which indicated that ecstasyinduced abnormalities are most prominent in basal ganglia and certain cortical areas; ecstasy-induced abnormalities in white matter were rarely reported and thus not expected. As cortical grey matter has very low anisotropy, it is very difficult to get reliable fractional anisotropy and apparent diffusion coefficient measurements in cortical areas. For this reason, only ROIs in white matter and basal ganglia were taken into account in the measurements of fractional anisotropy and apparent diffusion coefficients. Within the ROIs, individual mean values of fractional anisotropy, apparent diffusion coefficient, and regional relative cerebral blood volume ( $\mathrm{rrCBV}$ ) were calculated. Values of fractional anisotropy, apparent diffusion coefficient and rrCBV from ROIs in left and right hemispheres were averaged.

\section{SPECT acquisition and post-processing}

\section{Acquisition}

In a subgroup of the population $(n=47)$ SPECT imaging was performed with the radioligand $\left[{ }^{123} \mathrm{I}\right] 2 \beta$-carbomethoxy-3 $\beta$ (4-iodophenyl)-tropane $\left(\left[{ }^{123} \mathrm{I}\right] \beta\right.$-CIT) that binds to serotonin transporters, dopamine transporters and, to a lesser extent, noradrenaline transporters. The procedure of radiosynthesis of $\left[{ }^{123} \mathrm{I}\right] \beta$-CIT and acquiring of SPECT images were the same as previously described. ${ }^{14}$ A bolus of approximately $110 \mathrm{MBq}$ (3 mCi) $\left[{ }^{123} \mathrm{I}\right] \beta$-CIT was injected intravenously and SPECT images were acquired $4 \mathrm{~h}$ after the injection, when stable specific uptake to serotonin transporters is expected to be reached. ${ }^{14}$

\section{Post-processing}

Attenuation correction of all images was performed as previously described. ${ }^{14}$ Images were reconstructed in 3D mode (www. neurophysics.com). All SPECT scans were registered (rigid body) to the $\mathrm{T}_{1}-3 \mathrm{D}$ MRI scans of the same participant using a software package developed for $3 \mathrm{D}$ and $4 \mathrm{D}$ registration of multiple scans for radiotherapy application. ${ }^{15}$ Next, the same program was used to register the individual MRI scans to the MNI152 brain using
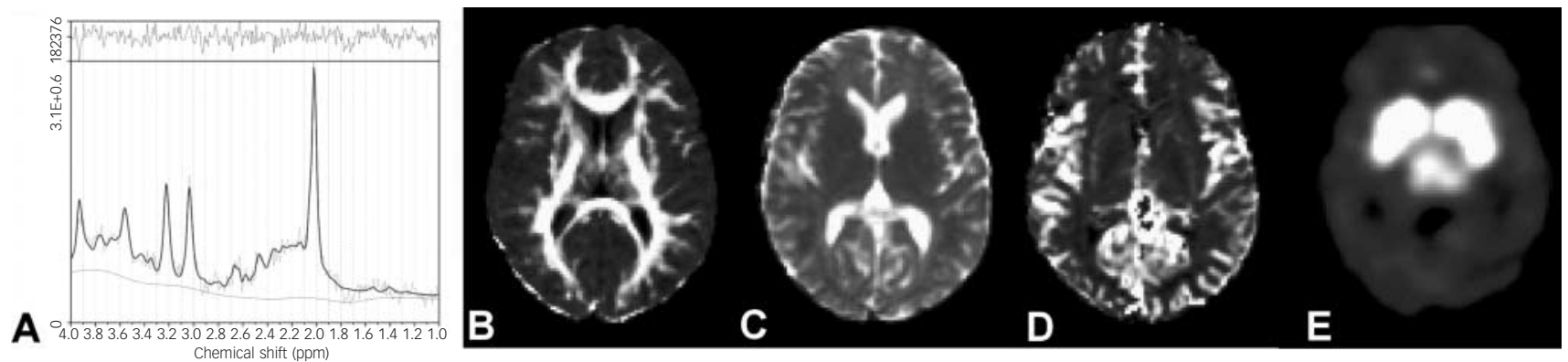

Fig. 1 Representative images of an individual (a) ${ }^{1} \mathrm{H}$-magnetic resonance spectroscopy after analysis by Linear Combination of Model spectra and representative (b) fractional anisotropy; (c) apparent diffusion coefficient; (d) regional relative cerebral blood volume; and (e) $\left.\left[{ }^{123}\right]\right] \beta-\mathrm{CIT}$ binding images after transformation to the spatially normalised Montreal Neurological Institute brain template. 


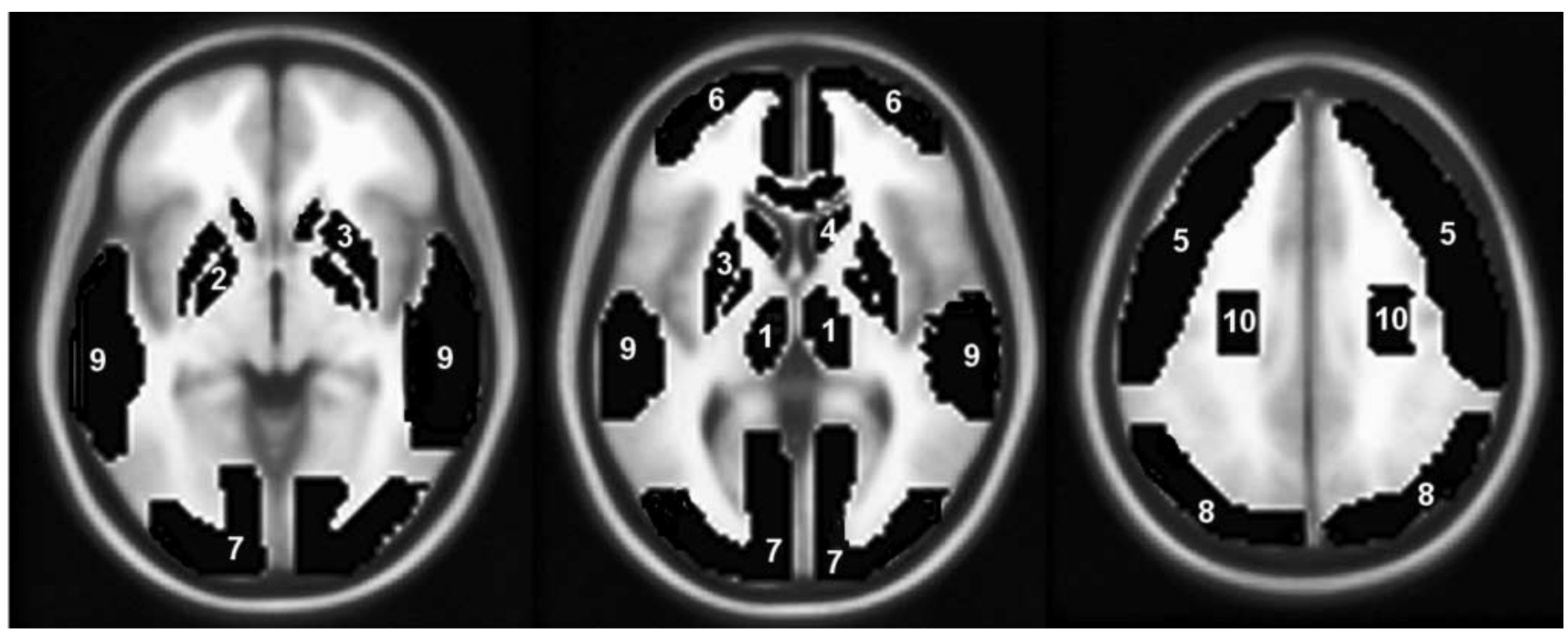

Fig. 2 Regions of interest used for analyses of diffusion tensor imaging and perfusion-weighted imaging scans drawn on magnetic resonance brain template at three levels.

1, thalamus; 2, globus pallidus; 3, putamen; 4, caudate nucleus; 5, dorsolateral frontal cortex; 6, mid-frontal cortex; 7, occipital cortex; 8, superior parietal cortex; 9, temporal cortex; 10 , white matter of the centrum semiovale.

affine transformations. For both registration steps an algorithm was used that maximises mutual information of the voxels of the scans to be registered. ${ }^{16}$ Finally, the software package was used to resample the individual SPECT images to the MNI152 brain (Fig.1), resulting in $91 \times 109 \times 92$ voxel images with voxel sizes of $2 \times 2 \times 2 \mathrm{~mm}^{3}$. In this way, all SPECT images could be analysed together.

For quantification, both $\mathrm{ROI}$ and voxel $\times$ voxel analyses were performed. For the ROI analysis, regions were drawn on the MNI152 template in midbrain, thalamus and temporal, frontal and occipital cortex. We did not measure serotonin transporter uptake in the putamen, caudate nucleus and globus pallidus, because there is no specific uptake to serotonin or dopamine transporters in these regions $4 \mathrm{~h}$ after $\left[{ }^{123} \mathrm{I}\right] \beta$-CIT injection. Activity in the cerebellum was assumed to represent non-displaceable activity (non-specific binding and free radioactivity). Specific to non-specific binding ratios were calculated as (activity in ROI activity in cerebellum)/ activity in cerebellum. The image registration was visually inspected to check its quality.

For the voxel $\times$ voxel analysis, the Statistical Parametric Mapping software (SPM2, Wellcome Department of Imaging Neuroscience, Functional Imaging Laboratory, London, UK; www.fil.ion.ucl.ac.uk/spm) was used. ${ }^{17}$ The registered scans were intensity-scaled to the corresponding mean cerebellar non-specific counts per voxel. The mean cerebellar counts were obtained from the ROI analysis. Then, smoothing was applied with SPM2 (Gaussian kernel with a $16 \mathrm{~mm}$ full width at half maximum) to reduce inter-individual anatomical differences that remained after stereotactical normalisation. $^{18}$

\section{Statistical analyses}

\section{Substance use variables and potential confounders}

Self-reported histories of drug use may not be fully accurate and there is variation in the amount of MDMA in different ecstasy tablets. In addition, drug use variables in the current study were not normally distributed, not even after log transformation. Therefore, drug use variables were dichotomised using cut-off scores, which were fixed to balance the distribution of users and non-users of a particular drug. For ecstasy, amphetamines and cocaine the cut-off score was arbitrarily determined at $>10$ tablets/occasions lifetime. For cannabis, the cut-off score was set higher ( $>50$ joints lifetime), because experimenting with cannabis is much more common than with other illicit drugs. Table 1 shows cut-off values, frequency distributions, means (s.d.) and medians for the substance variables in the total sample.

Phi coefficients were calculated to assess the associations between dichotomised drug-use and demographic variables (Table 2). The relatively low association between some independent variables does not affect the validity of the regression model, because each regressor is adjusted for the predictive effect of all other regressors in the model. The variance inflation factor was used to estimate multicollinearity. In the various analyses, variance inflation factor values ranged from 1.0 to 1.7 , indicating that factor correlations did not cause over-specification of the regression model, allowing for reliable estimation of the independent effects of the various drugs on the neuroimaging parameters.

Gender was included in all regression analyses because previous studies indicated that females are more vulnerable to the effects of ecstasy than males. ${ }^{1}$ The most important potential drug-use confounders amphetamine, cocaine and cannabis were included in all adjusted regression analyses. Additional confounders (age, verbal IQ, alcohol, tobacco) were chosen based on theoretical grounds per modality to reduce the number of regressors in the regression model: for ${ }^{1} \mathrm{H}-\mathrm{MRS}$, verbal IQ was added as an additional confounder because a relationship between brain metabolites and verbal IQ was reported; ${ }^{19}$ for diffusion tensor imaging no additional confounders were included in the analyses; and for perfusion-weighted and SPECT imaging, tobacco was added because previous studies showed a relationship between smoking and brain perfusion, ${ }^{20}$ as well as between smoking and serotonin transporter densities. ${ }^{21}$ Age was not included as a confounder, because of the relatively small age-range within the sample.

\section{Linear regression MRI and SPECT region of interest analyses}

To assess the specific effects of ecstasy and contributions of other drugs on the outcome parameters of MRI and SPECT imaging, linear multiple regression analyses were performed. Two different stepwise multiple linear regression models were used with imaging parameters as dependent variables. 


\begin{tabular}{|c|c|c|c|c|c|}
\hline Variable & Cut-off value & Participants, $n$ & Mean (s.d.) & Median & Range \\
\hline \multicolumn{6}{|l|}{ Gender } \\
\hline Male & & 44 & & & \\
\hline Female & & 27 & & & \\
\hline Age, years & & 71 & $23.3(3.8)$ & 22.6 & $18-37$ \\
\hline IQ (DART score) & & 71 & $101(7.7)$ & 100 & $83-122$ \\
\hline Ecstasy users & $>10$ tablets lifetime & 33 & $322(354)$ & 250 & $15-2000$ \\
\hline Amphetamine users & $>10$ occasions lifetime & 18 & 151 (154) & 120 & $15-600$ \\
\hline Cocaine users & $>10$ occasions lifetime & 22 & $72(70)$ & 43 & $12-300$ \\
\hline Cannabis users & $>50$ joints & 42 & $1234(1622)$ & 688 & $56-6650$ \\
\hline Alcohol users & $>10$ units per week & 36 & $22(12)$ & 22 & $12-60$ \\
\hline Tobacco users & $>10$ cigarettes per week & 32 & $85(46)$ & 80 & $17-200$ \\
\hline
\end{tabular}

Model 1 estimated the upper bound effect of ecstasy on outcome parameters, i.e. with adjustment for the effects of gender (and also verbal IQ in the case of ${ }^{1} \mathrm{H}-\mathrm{MRS}$ ), but without correction for the effects of other drugs. In the first step, gender (and IQ) was entered as the independent variable and in a second step, ecstasy was entered. The effect of ecstasy was quantified as the $R^{2}$-change between the first and the second steps of the model. This model resembles the approach in previous studies that compared ecstasy users with non-users. However, the effect of ecstasy in this model is likely to be an overestimation of the real independent effect of ecstasy owing to the lack of correction for the impact of other drugs on the imaging parameters of neurotoxicity.

Model 2 estimated the lower bound effect of ecstasy on outcome parameters after adjustment for the effects of gender, IQ and the use of substances other than ecstasy. In analogy to model 1, first gender (and IQ in the case of ${ }^{1} \mathrm{H}-\mathrm{MRS}$ ) and substance use other than ecstasy (cannabis, amphetamines and cocaine in all analyses and tobacco in the case of perfusionweighted imaging and SPECT) were entered in the model as independent variables. In a second step, ecstasy was entered as an additional independent variable. Similar to model 1 , the independent effect of ecstasy use was quantified as the $R^{2}$-change between the first and second steps of the model. The effect of ecstasy in model 2 is presumably an underestimation of the real independent effect of ecstasy, because of possible over-correction for the effects of other drugs.

Linear regression analyses were performed using SPSS version 11.5 for Windows. $P$ values $<0.05$ were considered statistically significant. Besides $R^{2}$, unstandardised regression coefficients $(B)$ were used to reflect the predictive power of the different regressors. In the online Table DS1, $B$ values are reported with $95 \%$ confidence intervals $(95 \% \mathrm{CI})$ and in the text with their two-tailed significance level ( $P$ value).

\section{SPECT voxel $\times$ voxel analysis}

For the voxel $\times$ voxel analyses we did not use the sample as a whole as in the other analyses (because in SPM it was not possible to perform similar voxel-based linear regression analyses in the sample as a whole as for the ROI analyses), but divided the sample $(n=47)$ into five groups, based upon the dichotomised drug-use variables (Table 1). The groups included: heavy ecstasy polydrug users $(n=10)$; selective ecstasy and cannabis users $(n=4)$; ecstasynaïve polydrug (amphetamine and/or cocaine and cannabis) users $(n=5)$; ecstasy-naïve cannabis users $(n=16)$; and drug-naïve controls $(n=12)$. The $\left[{ }^{123} \mathrm{I}\right] \beta$-CIT binding ratios of the stereotactically and intensity-normalised and smoothed SPECT images were compared between the five groups on a voxel $\times$ voxel basis by means of the spatial extent statistical theory using SPM2. ${ }^{17,18}$ The positron emission tomography (PET)/SPECT model 'single subject conditions and covariates' was chosen. Five conditions and no covariates were included. The main comparison was between ecstasy users and non-users (groups 1 and $2 v$. groups 3,4 and 5). Because this showed some significant clusters, post hoc comparisons were made between the different groups to analyse whether significant differences were caused by ecstasy or by other substances. An effect was considered statistically significant if a cluster of at least 20 connected voxels reached a $P$ value $<0.001$ (one-sided; $T=3.30$, uncorrected for multiple

Table 2 Phi correlations between dichotomised substance use variables in the whole sample ${ }^{a}(n=71)$

\begin{tabular}{|c|c|c|c|c|c|c|c|c|}
\hline Age & Gender & DART IQ & Alcohol & Tobacco & Ecstasy & Amphetamine & Cocaine & Cannabis \\
\hline Age & NS & NS & NS & NS & NS & NS & NS & NS \\
\hline Gender & & NS & NS & NS & NS & NS & NS & NS \\
\hline DART IQ & & & NS & NS & NS & NS & NS & -0.23 \\
\hline Alcohol & & & & NS & NS & NS & NS & 0.38 \\
\hline Tobacco & & & & & 0.40 & NS & 0.31 & 0.41 \\
\hline Ecstasy & & & & & & 0.43 & 0.54 & NS \\
\hline Amphetamine & & & & & & & 0.45 & NS \\
\hline Cocaine & & & & & & & & NS \\
\hline Cannabis & & & & & & & & NS \\
\hline
\end{tabular}


comparisons). Clusters of voxels surviving the thresholds were colour-coded and superimposed on the MNI152 template.

\section{Results}

\section{Sample characteristics and substance use}

Characteristics of demography and substance use of the total sample are presented in Table 1. Mean cumulative dose of ecstasy within the ecstasy group was 322 tablets $($ s.d.=354). Mean time since last ecstasy use within this group was 8.2 weeks (s.d.=9.8), age at first use 17.7 years (s.d.=2.8) and usual ecstasy dose was 2.7 tablets per session (s.d.=1.6).

\section{${ }^{1} \mathrm{H}-\mathrm{MRS}$, diffusion tensor imaging and perfusion-weighted imaging}

Two participants had enlarged lateral ventricles (one ecstasy-naïve cannabis user and one ecstasy polydrug user), hampering matching to the MNI template, so the diffusion tensor imaging and perfusion-weighted imaging of these people were not included. Owing to technical problems, ${ }^{1} \mathrm{H}$-MRS was not performed in two individuals and diffusion tensor imaging in one person. Therefore, we report measurements of ${ }^{1} \mathrm{H}-\mathrm{MRS}$ and $\mathrm{rrCBV}$ in 69 participants and of fractional anisotropy and apparent diffusion coefficient in 68 participants.

Online Table DS1 shows results from the linear regression analyses. There was no significant effect of ecstasy use on the brain metabolites ratios $\mathrm{NAA} / \mathrm{Cr}, \mathrm{Cho} / \mathrm{Cr}$ and $\mathrm{mI} / \mathrm{Cr}$ in any of the three regions. With diffusion tensor imaging no significant effects of ecstasy on apparent diffusion coefficient in basal ganglia were observed, but ecstasy did have a significant negative effect on fractional anisotropy in the thalamus (model $1: R_{\text {ecstasy }}^{2}=16.6 \%$; $\left.B_{\text {ecstasy }}=-20.09, P<0.001\right)$. After adjusting for other drugs, the negative effect of ecstasy on thalamic fractional anisotropy remained significant (model 2: $R_{\text {ecstasy }}^{2}=9.7 \% ; B_{\text {ecstasy }}=-18.76$, $P=0.006$ ). Also, gender had a significant effect on fractional anisotropy in the thalamus (lower in females) ( $B_{\text {gender }}=-11.95$, $P=0.043)$. Ecstasy had a significant positive effect on $\mathrm{rCBV}$ in the thalamus (model 1: $R_{\text {ecstasy }}^{2}=7.3 \% ; B_{\text {ecstasy }}=0.094, P=0.024$ ) and the temporal cortex (model $1: R_{\text {ecstasy }}^{2}=8.1 \% ; B=0.111$, $P=0.018$ ). These effects remained statistically significant after correction for other substances (model 2: $R_{\text {ecstasy }}^{2}=6.4 \%$; $B_{\text {ecstasy }}=0.114, P=0.037$ for the thalamus and $R_{\text {ecstasy }}^{2}=6.8 \%$; $B_{\text {ecstasy }}=0.131, P=0.030$ for the temporal cortex).

According to model 2, amphetamine had a positive effect on mid-occipital $\mathrm{mI} / \mathrm{Cr}$ ratios $\left(B_{\text {amphetamine }}=0.085, P=0.031\right.$ ), a negative effect on fractional anisotropy in the centrum semiovale ( $B_{\text {amphetamine }}=-24.53, P=0.033$ ) and a negative effect on $\mathrm{rCBV}$ in the superior parietal cortex $\left(B_{\text {amphetamine }}=-0.109, P=0.038\right)$. Use of cocaine had a positive effect on both $\mathrm{Cho} / \mathrm{Cr}\left(B_{\text {cocaine }}=0.027\right.$, $P=0.030)$ and $\mathrm{mI} / \mathrm{Cr}\left(B_{\text {cocaine }}=0.144, P=0.004\right)$ ratios in the centrum semiovale, whereas cocaine had a negative effect on $\mathrm{Cho} / \mathrm{Cr}$ in the mid-frontal cortex $\left(B_{\text {cocaine }}=-0.027, P=0.036\right)$. Cannabis did not have any significant effect on magnetic resonance outcome parameters.

\section{[ $\left.{ }^{123} 1\right] \beta-C I T$ SPECT}

Region of interest analyses showed a significant negative effect of ecstasy on $\left[{ }^{123} \mathrm{I}\right] \beta$-CIT binding in the thalamus (model 1 : $R_{\text {ecstasy }}^{2}=31.0 \% ; B_{\text {ecstasy }}=-0.394, P<0.001$ ), frontal cortex (model $\left.1: R_{\text {ecstasy }}^{2}=16.4 \% ; B_{\text {ecstasy }}=-0.090, P=0.005\right)$ and temporal cortex (model 1: $R_{\text {ecstasy }}^{2}=21.1 \% ; \quad B_{\text {ecstasy }}=-0.160, P=0.001$ ) (online Table DS1). After adjustment for amphetamines, cocaine, cannabis and tobacco (model 2), the effect remained significant in the thalamus $\left(R_{\text {ecstasy }}^{2}=15.2 \% ; B_{\text {ecstasy }}=-0.343, P=0.003\right)$, but not in the frontal and temporal cortex $(P=0.140$ and $P=0.076$ respectively). Amphetamine, cocaine and cannabis use did not have significant effects on $\left[{ }^{123} \mathrm{I}\right] \beta$-CIT binding in any of the ROIs.

Also with voxel $\times$ voxel analysis, lower $\left[{ }^{123} \mathrm{I}\right] \beta$-CIT binding was observed in the thalamus of ecstasy users compared with non-users $\left(Z_{\max }=5.07, P_{\text {corrected, cluster-level }}=0.001\right.$; coordinates of the highest $Z$-value: $2,-22,8$ ) (online Fig. DS1). The degree of $\left[{ }^{123} \mathrm{I}\right] \beta$-CIT binding in the cingulate gyrus was also significantly lower in ecstasy users than in non-users, although this should be interpreted with caution, because the highest $Z$-value was exactly in the midline $\left(Z_{\max }=4.15, \quad P_{\text {corrected, cluster-level }}<0.001\right.$; coordinates of the highest $Z$-value: $0,42,8)$. Post hoc, the same cluster of significantly lower $\left[{ }^{123} \mathrm{I}\right] \beta$-CIT binding in the thalamus was observed in ecstasy users when we compared ecstasy users with substance-using controls (groups 1 and $2 v$. groups 3 and 4 ), when we compared ecstasy polydrug users with ecstasy-naïve polydrug users (group $1 v$. group 3), and when we compared selective ecstasy and cannabis users with ecstasy-naïve cannabis users (group $2 v$. group 4). The cluster of significantly lower $\left[{ }^{123} \mathrm{I}\right] \beta$-CIT binding in the anterior cingulate gyrus was observed in ecstasy users when we compared ecstasy users with substanceusing controls (groups 1 and $2 v$. groups 3 and 4 ) and when we compared selective ecstasy and cannabis users with ecstasy-naïve cannabis users (group $2 v$. group 4 ), but not when we compared ecstasy polydrug users with ecstasy-naïve polydrug users (group $1 v$. group 3). No clusters of increased $\left[{ }^{123} \mathrm{I}\right] \beta$-CIT binding were observed in ecstasy users in any of the comparisons. Selective ecstasy and cannabis users did not have clusters of significantly different $\left[{ }^{123} \mathrm{I}\right] \beta$-CIT binding than ecstasy polydrug users (group 1 v. group 2). Cannabis users did not significantly differ from drug-naïve controls (group $4 v$. group 5), and ecstasy-naïve polydrug users did not differ on $\left[{ }^{123} \mathrm{I}\right] \beta$-CIT binding from drugnaïe controls (group $3 v$. group 5).

\section{Discussion}

Use of the party drug ecstasy has been associated with decreased serotonergic function as shown by decreased densities of serotonin transporters in membranes of serotonin axons, decreased neurocognitive performance, and increased depression scores in ecstasy users. ${ }^{1,2}$ The loss of transporters in serotonergic terminals most likely represents axonal injury, since preclinical studies show that ecstasy typically induces axonal injury/loss of serotonergic cells and that the serotonergic cell bodies remain intact. ${ }^{22}$ However, the validity of findings suggesting ecstasy-related neurotoxicity in humans is debated because most studies have methodological limitations, including inadequate control of potential confounders such as polydrug use. The present study was designed to overcome limitations of previous studies, by adequately controlling for polydrug use and by combining, for the first time, advanced magnetic resonance and SPECT imaging techniques in the same sample to study different aspects of brain involvement.

\section{Polydrug confounding in human ecstasy studies}

Because almost all ecstasy users are polydrug users ${ }^{3}$ it is difficult to differentiate effects of ecstasy from potential effects of other psychoactive drugs. Some studies reported that signs of neurotoxicity in ecstasy users might be related not to ecstasy use alone but rather to polydrug use or the use of other psychoactive drugs such as cannabis, amphetamines or cocaine. ${ }^{23}$ Only some of the previous studies adequately controlled for use of drugs other than ecstasy by including a group of 'pure' ecstasy users, ${ }^{24}$ by including a drug-using but ecstasy-naive control group ${ }^{25}$ or by statistically 
adjusting for polydrug use. ${ }^{26}$ However, these attempts have limitations because 'pure' ecstasy users are very rare $^{3}$ and drug use in the control groups was generally lower than in the ecstasy groups and mainly comprised the use of cannabis and much less the use of amphetamines and cocaine. Controlling for polydrug use in a statistical regression analysis was generally hampered by the fact that cannabis, cocaine and amphetamine use were almost always strongly correlated with ecstasy use, leading to multicollinearity and the impossibility of statistical adjustment for these potential confounders in multiple regression analysis. ${ }^{26}$

In our study we used a new approach by including a carefully selected sample of drug users with specific variations in amount and type of drugs used. This strategy successfully reduced the magnitude of the correlations between ecstasy use and the use of other substances, and allowed us to use linear multiple regression analysis to differentiate between the effects of ecstasy and of other substances.

\section{Specific effects of ecstasy on the thalamus}

The most interesting finding is that different imaging techniques all showed a specific effect of ecstasy on the thalamus. Even after adjustment for amphetamine, cocaine, cannabis and other relevant potential confounders, a significant effect of ecstasy, and no effects of any of the other drugs, was found on $\left[{ }^{123} \mathrm{I}\right] \beta$-CIT binding (reduced), fractional anisotropy (reduced) and $\mathrm{rrCBV}$ (increased) in the thalamus. As $\left[{ }^{123} \mathrm{I}\right] \beta$-CIT SPECT was previously validated to assess in vivo binding to serotonin transporters, the finding of decreased $\left[{ }^{123} \mathrm{I}\right] \beta$-CIT binding probably reflects lower serotonin transporter densities in ecstasy users. ${ }^{14,27}$ Moreover, the thalamus is a serotonin transporter-rich area and previous studies showed that $\left[{ }^{123} \mathrm{I}\right] \beta$-CIT binding in the thalamus is mainly related to transporter binding, although the thalamus also contains noradrenaline transporters. Diffusion tensor imaging measures diffusional motion of water molecules in the brain which is normally restricted in amplitude and direction by cellular structures such as axons. ${ }^{28}$ When axons are damaged, extracellular water content increases and fractional anisotropy decreases. Therefore, it is likely that the observed decreased fractional anisotropy is related to ecstasy-induced axonal injury. An alternative explanation could be that decreased fractional anisotropy relates to increased brain perfusion in the thalamus, which also gives an increase in extracellular water content. As ecstasy was previously shown to reduce extracellular serotonin and serotonin is involved in regulation of brain microcirculation, mainly as a vasoconstrictor, ${ }^{29}$ ecstasy-induced serotonin depletion may have led to vasodilatation and the observed increase in $\operatorname{rCBV}$. Taken together, it seems that these measurements in the thalamus converge in the direction of decreased serotonergic function, with decreased serotonin transporter binding and decreased fractional anisotropy values probably reflecting damage to serotonergic axons and increased rrCBV due to decreased vasoconstriction caused by depletion of serotonin. Previous studies in animals also showed ecstasy-induced axonal damage to the serotonergic axons of the thalamus, although signs of re-innervation after a period of recovery were also observed. ${ }^{30}$ As the thalamus plays a key role in awareness, attention and neurocognitive processes such as memory and language, ${ }^{31}$ one can speculate that ecstasy-induced serotonergic damage to the thalamus is (partly) responsible for reduced verbal memory performance frequently reported in ecstasy users.

\section{Integration with prior SPECT/PET studies}

Previous imaging studies in ecstasy users mainly used PET or SPECT techniques with tracers that bind to the serotonin transporter. ${ }^{1}$ In line with the current study, almost all of these studies reported decreased binding in the thalamus of ecstasy users. However, most of these studies also reported lower serotonin transporter-binding in other subcortical and cortical areas, although these areas varied in different studies. When only adjusted for gender and not for other substances, we also observed lower $\left[{ }^{123} \mathrm{I}\right] \beta$-CIT binding in ecstasy users in the frontal cortex, mainly located in the anterior cingulate gyrus as shown by the voxel $\times$ voxel analysis, and temporal cortex. However, decreased $\left[{ }^{123} \mathrm{I}\right] \beta$-CIT binding in these areas seemed to be related to polydrug use in general, and not to ecstasy or any other drug in particular, because none of the psychoactive substances was a significant predictor in the adjusted model. Moreover, decreased $\left[{ }^{123} \mathrm{I}\right] \beta$-CIT binding ratios in areas with few serotonin transporters, such as the cortical areas, should be interpreted with caution. ${ }^{14}$ We did not observe decreased $\left[{ }^{123} \mathrm{I}\right] \beta$-CIT binding in midbrain and occipital cortex as previously observed and we could not reproduce findings that women might be more susceptible than men to the effects of ecstasy on the serotonergic system. ${ }^{1,2}$ A recent PET study in patients who had previously been treated with the appetite suppressants fenfluramine and dexfenfluramine (known serotonergic neurotoxins in animals) also showed reductions in serotonin transporters, and these reductions were greatest in the thalamus. ${ }^{32}$ This finding is of particular interest since these patients had no or low exposure to drugs of misuse.

\section{Integration with prior magnetic resonance studies}

Only few previous studies used advanced magnetic resonance techniques to assess ecstasy-induced neurotoxicity. One preliminary study measured apparent diffusion coefficients in ecstasy users, although not in the thalamus, and reported an increased apparent diffusion coefficient in the globus pallidus of ecstasy users, suggesting axonal damage. ${ }^{33}$ In our study we did not find any effect of ecstasy on apparent diffusion coefficient measurements, as would be expected, especially because we did find a decrease in fractional anistropy, which is often related to an increase in apparent diffusion coefficient. The same study of Reneman et $^{3 l^{33}}$ (not including measurements in the thalamus) also examined brain perfusion and showed increased rrCBV values in the globus pallidus of ecstasy users. Another study by our group reported increased rrCBV values in the globus pallidus and thalamus of two former ecstasy users who had been abstinent for 18 weeks on average. ${ }^{34}$ In our study we did not observe increased rrCBV values in the globus pallidus. However, we observed an increase in rrCBV, only related to ecstasy and not to other drugs, in the thalamus and also in the temporal cortex, an area that was not included in the previous studies. Cerebrovascular changes in ecstasy users were also observed in a previous SPECT study, measuring regional cerebral blood flow. ${ }^{10}$

With ${ }^{1} \mathrm{H}-\mathrm{MRS}$, we did not find indications of neuronal damage (i.e. no decrease in NAA/Cr ratios and no increase in $\mathrm{Cho} / \mathrm{Cr}$ and $\mathrm{mI} / \mathrm{Cr}$ ratios in ecstasy users. However, we did not perform ${ }^{1} \mathrm{H}$-MRS in the thalamus, because it is technically difficult to obtain reliable ${ }^{1} \mathrm{H}$-MRS measurements in that area owing to magnetic field inhomogeneities and partial volume effects. Previous studies showed lower NAA/Cr ratios in the frontal cortex of ecstasy users with an average cumulated dose of more than 700 tablets, probably reflecting neuronal loss, whereas others found no difference in NAA/Cr ratios in cortical brain regions in individuals with more moderate lifetime doses. ${ }^{1}$ Therefore, these effects may only become apparent after very heavy ecstasy (polydrug) use. On the other hand, a recent experimental study in non-human primates observed reductions in NAA in the hypothalamus even after low MDMA exposure. ${ }^{35}$ 
An increased myoinositol in parietal white matter was observed in only one study. ${ }^{1}$

\section{Effects of other drugs}

In addition to the effects of ecstasy, the current study design enabled us to explore effects of other substances on the outcome parameters. Amphetamine use, mainly D-amphetamine in The Netherlands, also showed some significant effects on the outcome parameters. However, the different imaging techniques showed effects of amphetamine in different brain areas, and therefore these findings are less consistent than the converging findings of the ecstasy effects on the thalamus. Amphetamine users showed an increased $\mathrm{mI} / \mathrm{Cr}$ ratio in the mid-occipital grey matter and decreased fractional anisotropy in the centrum semiovale, and decreased rrCBV in the superior parietal grey matter. As it is known that $\mathrm{D}$-amphetamine use is mainly associated with dopaminergic toxicity, ${ }^{36}$ these effects may be related to damage of the dopaminergic system. Cocaine had a positive effect on Cho/ $\mathrm{Cr}$ and $\mathrm{mI} / \mathrm{Cr}$ ratios in the centrum semiovale, which might be related to increased glial activation. In contrast, cocaine had a negative effect on the $\mathrm{Cho} / \mathrm{Cr}$ ratio in the mid-frontal grey matter. A previous study of cocaine users showed increased $\mathrm{mI} / \mathrm{Cr}$ ratios in both frontal grey and white matter, as well as a decreased NAA/Cr ratio in the frontal cortex. ${ }^{37}$ Cocaine did not have any significant effect on outcomes of diffusion tensor imaging, perfusionweighted imaging or SPECT measurements. Cannabis use had no significant effect on any of the outcome parameters. Also, other studies showed little evidence that chronic cannabis use causes permanent brain damage ${ }^{38}$ or changes in cerebral blood flow, ${ }^{39}$ although there are indications that mild cognitive impairment can occur in very heavy chronic cannabis use. ${ }^{40}$

\section{Limitations}

Owing to its cross-sectional design and lack of baseline data it is difficult to draw firm conclusions regarding the causality of the observed relationships between ecstasy use and the neuroimaging outcome parameters, because it is possible that differences between ecstasy users and controls were pre-existent. We had to rely on the retrospective self-reported records of drug use in the past using drug-history questionnaires of which the reliability is uncertain. Hair analyses supported the plausibility of selfreported data on ecstasy use in our study, although it yields no information on patterns of ecstasy use, i.e. frequency, dosage or cumulative lifetime dose. There will also have been variation in dosage and purity of ecstasy tablets, although pill-testing confirms that in The Netherlands $95 \%$ of the tablets sold as ecstasy contain MDMA as a major component, as previously discussed. ${ }^{4}$ Also, environmental circumstances under which ecstasy was taken and simultaneous use of other substances were heterogeneous. Because of our recruitment strategy, the current sample cannot be regarded as representative of all heavy ecstasy users. Therefore, the point estimate of the effect of ecstasy on the neurotoxicity parameters should be interpreted with caution. More important, the specific recruitment strategy allowed us to test whether the observed neurotoxic effect of ecstasy remained significant after statistical control for the use of other drugs such as cannabis, amphetamines and cocaine. Although we succeeded in creating relatively independent factors for ecstasy and cannabis use, correlations between use of ecstasy and amphetamine and cocaine were relatively low but still substantial and statistically significant. None the less, correlations between use of ecstasy and other illicit drugs were lower than usually found after random recruitment among frequent ecstasy users $^{41}$ and statistical collinearity analyses did not suggest any problems of multicollinearity, indicating that the regression model allowed for reliable estimation of the effects of the various drugs. Moreover, the association between ecstasy use and its most commonly used co-drug, cannabis, was successfully removed as a result of sample stratification, thereby controlling for an important confounder. To prevent measuring acute pharmacological effects, participants had to abstain from psychoactive drugs for 2 weeks before examinations. This may have led to some inevitable selection, especially among heavy cannabis users. Finally, we did not correct for multiple comparisons in order to minimise the risk of falsenegative results (type II errors). ${ }^{42}$ The use of various imaging techniques and assessments in different brain regions may have introduced some false-positive findings (type I errors).

\section{Maartje M. L. de Win, MD, PhD, Department of Radiology, Academic Medical Center, University of Amsterdam, and The Netherlands and Graduate School of Neurosciences, Amsterdam; Gerry Jager, PhD, Rudolf Magnus Institute of Neurosciences, Amsterdam; Gerry Jager, PhD, Rudolf Magnus Institute of
Neuroscience, Department of Psychiatry, University Medical Center, Utrecht; Jan Booij, MD, PhD, Department of Nuclear Medicine, Academic Medical Center, University of Amsterdam; Liesbeth Reneman, MD, PhD, Department of Radiology, Academic Medical Center, University of Amsterdam; Thelma Schilt, MSc, Amsterdam Institute for Addiction Research and Department of Psychiatry, Academic Medical Center, University of Amsterdam; Cristina Lavini, MPhil, Department of Radiology, Academic Medical Center, University of Amsterdam; Sílvia D. Olabarriaga, PhD, Informatics Institute, University of Amsterdam; Nick F. Ramsey, PhD, Rudolf Magnus Institute of Neuroscience, Department of Neurosurgery, University Medical Center, Utrecht; Gerard J. den Heeten, MD, PhD Department of Radiology, Academic Medical Center, University of Amsterdam; Wim van den Brink, MD, PhD, Amsterdam Institute for Addiction Research and Department of Psychiatry, Academic Medical Center, University of Amsterdam, The Netherlands}

Correspondence: Maartje M.L. de Win, Department of Radiology, G1-229, University of Amsterdam, Academic Medical Center, Meibergdreef 9, $1105 \mathrm{AZ}$ Amsterdam, The Netherlands. Email: m.m.dewin@amc.uva.nl

First received 21 Jan 2007, final revision 29 Jan 2008, accepted 11 Feb 2008

\section{Acknowledgements}

The NeXT study was supported by a grant of The Netherlands Organization for Health Research and Development as part of their Program Addiction (ZonMw 310-00-036). S.D.O. participates in the Virtual Laboratory for e-Science project, which is supported by a BSIK grant from the Dutch Ministry of Education, Culture and Science. We thank Professor M. Moseley (Lucas MRS Center, Stanford University, USA) for support in the implementation of the diffusion tensor imaging protocol. We also thank Dirk Korf for his help on the design of the study; Hylke Vervaeke for recruiting volunteers; Sarah Dijkink and Ivo Bisschops for assistance with data collection; Jacco Visser and other technicians for making the SPECT scans; Benoit Faivre, Dick Veltman, Matthan Caan, Frans Vos, Marcel van Herk and Jan Habraken for their help in analysing the SPECT data; Erik-Jan Vlieger and van Herk and Jan Habraken for their help in analysing the SPECT data; Erik-Jan Vlieger and
Jeroen snel for their help on the post-processing of the diffusion tensor imaging and perfusion-weighted imaging scans; Charles Majoie for reading all anatomical magnetic resonance scans; and Maarten Koeter and Ben Schmand for their advice on the statistical analysis.

\section{References}

1 Reneman L, de Win MML, van den Brink W, Booij J, den Heeten GJ. Neuroimaging findings with MDMA/ecstasy: technical aspects, conceptual issues and future prospects. J Psychopharmacol 2006; 20: 164-75.

2 Parrott AC. Human psychopharmacology of Ecstasy (MDMA): a review of 15 years of empirical research. Hum Psychopharmacol 2001; 16: 557-77.

3 Schuster P, Lieb R, Lamertz C, Wittchen HU. Is the use of ecstasy and hallucinogens increasing? Results from a community study. Eur Addict Res 1998; 4: 75-82.

4 de Win MML, Jager G, Vervaeke H, Schilt T, Reneman L, Booij J, Verhulst FC, den Heeten GJ, Ramsey NF, Korf DJ, van den Brink W. The Netherlands XTC Toxicity (NeXT) study: objectives and methods of a study investigating causality, course, and clinical relevance. Int J Methods Psychiatr Res 2005; 14: $167-85$.

5 Jager G, de Win MML, van der Tweel I, Schilt T, Kahn RS, van den Brink W, van Ree JM, Ramsey NF. Assessment of cognitive brain function in ecstasy users and contributions of other drugs of abuse: results from an FMRI study. Neuropsychopharmacology 2008; 33: 247-58. 
6 Schilt T, Win MM, Jager G, Koeter MW, Ramsey NF, Schmand B, van den Brink W. Specific effects of ecstasy and other illicit drugs on cognition in poly-substance users. Psychol Med 2007; 8: 1-9.

7 Van Vliet I, Leroy H, van Megen H. M.I.N.I. Internationaal Neuropsychiatric Interview (Dutch version). Leiden University Medical Center, 2000.

8 Nelson HE. The Revised National Adult Reading Test Manual. nferNelson, 1991.

9 de Win MML, Reneman L, Jager G, Vlieger EJP, Olabarriaga SD, Lavini C Bisschops I, Majoie CBLM, Booij J, den Heeten GJ, van den Brink W. A prospective cohort study on sustained effects of low-dose ecstasy use on the brain in new ecstasy users. Neuropsychopharmacology 2007; 32: 45870.

10 Chang L, Grob CS, Ernst T, Itti L, Mishkin FS, Jose-Melchor R, Poland RE. Effect of ecstasy [3,4-methylenedioxymethamphetamine (MDMA)] on cerebra blood flow: a co-registered SPECT and MRI study. Psychiatry Res 2000; 98: 15-28.

11 Reneman $L$, Majoie $C B$, Flick $H$, den Heeten GJ. Reduced N-acetylaspartate levels in the frontal cortex of 3,4-methylenedioxymethamphetamine (Ecstasy) users: preliminary results. AJNR Am J Neuroradiol 2002; 23: 231-7.

12 Provencher SW. Estimation of metabolite concentrations from localized in vivo proton NMR spectra. Magn Reson Med 1993; 30: 672-9.

13 Hunsche S, Moseley ME, Stoeter P, Hedehus M. Diffusion-tensor MR imaging at 1.5 and 3.0 T: initial observations. Radiology 2001; 221: 550-6.

14 de Win MML, Habraken JBA, Reneman L, van den Brink W, den Heeten GJ, Booij J. Validation of $\left[{ }^{123} 1\right] \beta$-CIT SPECT to assess serotonin transporters in vivo in humans: a double-blind, placebo-controlled, crossover study with the selective serotonin reuptake inhibitor citalopram. Neuropsychopharmacology 2005; 30: 996-1005.

15 Wolthaus JW, van Herk M, Muller SH, Belderbos JS, Lebesque JV, de Bois JA Rossi MM, Damen EM. Fusion of respiration-correlated PET and CT scans: correlated lung tumour motion in anatomical and functional scans. Phys Med Biol 2005; 50: 1569-83.

16 Maes F, Collignon A, Vandermeulen D, Marchal G, Suetens P. Multimodality image registration by maximization of mutual information. IEEE Trans Med Imaging 1997; 16: 187-98.

17 Friston KJ, Holmes AP, Worsley KJ, Poline JB, Frith C, Frackowial RSJ. Statistical parametric maps in functional imaging: a general linear approach Hum Brain Mapp 1995; 2: 189-210.

18 Worsley KJ, Marrett S, Neelin P, Evans AC. Searching scale space for activation in PET images. Hum Brain Mapp 1996; 4: 74-90.

19 Jung RE, Brooks WM, Yeo RA, Chiulli SJ, Weers DC, Sibbitt WL, Jr. Biochemica markers of intelligence: a proton MR spectroscopy study of normal human brain. Proc Biol Sci 1999; 266: 1375-9.

20 Zubieta JK, Heitzeg MM, Xu Y, Koeppe RA, Ni L, Guthrie S, Domino EF. Regional cerebral blood flow responses to smoking in tobacco smokers after overnight abstinence. Am J Psychiatry 2005; 162: 567-77.

21 Staley JK, Krishnan-Sarin S, Zoghbi S, Tamagnan G, Fujita M, Seibyl JP, Maciejewski PK, O'Malley S, Innis RB. Sex differences in $\left[{ }^{123} 1\right] \beta-C I T$ SPECT measures of dopamine and serotonin transporter availability in healthy smokers and nonsmokers. Synapse 2001; 41: 275-84.

22 Hatzidimitriou G, McCann UD, Ricaurte GA. Altered serotonin innervation patterns in the forebrain of monkeys treated with $(+/-) 3,4-$ methylenedioxymethamphetamine seven years previously: Factors influencing abnormal recovery. J Neurosci 1999; 19: 5096-107.

23 Gouzoulis-Mayfrank E, Daumann J. The confounding problem of polydrug use in recreational ecstasy/MDMA users: a brief overview. J Psychopharmacol 2006; 20: 188-93.
24 Halpern JH, Pope HG, Jr, Sherwood AR, Barry S, Hudson Jl, Yurgelun-Todd D. Residual neuropsychological effects of illicit 3,4-methylenedioxymethamphetamine (MDMA) in individuals with minimal exposure to other drugs. Drug Alcohol Depend 2004; 75: 135-47.

25 Montgomery C, Fisk JE, Newcombe R, Murphy PN. The differential effects of ecstasy/polydrug use on executive components: shifting, inhibition, updating and access to semantic memory. Psychopharmacology (Berl) 2005; 182 262-76.

26 Verkes RJ, Gijsman HJ, Pieters MS, Schoemaker RC, de Visser S, Kuijpers M, Pennings EJ, de Bruin D, Van de Wijngaart G, van Gerven JM, Cohen AF. Cognitive performance and serotonergic function in users of ecstasy. Psychopharmacology (Berl) 2001; 153: 196-202.

27 de Win MML, de Jeu RAM, de Bruin K, Habraken JBA, Reneman L, Booij J, den Heeten GJ. Validity of in vivo $\left.\left[{ }^{123}\right]\right] \beta$-CIT SPECT in detecting MDMA-induced neurotoxicity in rats. Eur Neuropsychopharmacol 2004; 14: 185-9.

28 Le Bihan D, Turner R, Douek P, Patronas N. Diffusion MR imaging: clinical applications. AJR Am J Roentgenol 1992; 159: 591-9.

29 Cohen Z, Bonvento G, Lacombe $P$, Hamel E. Serotonin in the regulation of brain microcirculation. Prog Neurobiol 1996; 50: 335-62.

30 Ricaurte GA, Martello AL, Katz JL, Martello MB. Lasting effects of (+-)-3,4methylenedioxymethamphetamine (MDMA) on central serotonergic neurons in nonhuman primates: neurochemical observations. J Pharmacol Exp Ther 1992; 261: 616-22.

31 Herrero MT, Barcia C, Navarro JM. Functional anatomy of thalamus and basal ganglia. Childs Nerv Syst 2002; 18: 386-404.

32 McCann UD, Szabo Z, Vranesic M, Seckin E, Wand G, Duval A, Dannals RF, Ricaurte GA. Quantitative positron emission tomography studies of the serotonin transporter in humans previously treated with the appetite suppressants fenfluramine or dexfenfluramine. Mol Imaging Biol 2007; 9: $151-7$.

33 Reneman L, Majoie CB, Habraken JB, den Heeten GJ. Effects of ecstasy (MDMA) on the brain in abstinent users: initial observations with diffusion and perfusion MR imaging. Radiology 2001; 220: 611-7.

34 Reneman L, Habraken JB, Majoie CB, Booij J, den Heeten GJ. MDMA ('ecstasy') and its association with cerebrovascular accidents: preliminary findings. AJNR Am J Neuroradiol 2000; 21: 1001-7.

35 Meyer JS, Brevard ME, Piper BJ, Ali SF, Ferris CF. Neural effects of MDMA as determined by functional magnetic resonance imaging and magnetic resonance spectroscopy in awake marmoset monkeys. Ann $N$ Y Acad Sci 2006; 1074: 365-76.

36 Booij J, de Bruin K, Gunning WB. Repeated administration of d-amphetamine induces loss of [ ${ }^{123}$ I]FP-CIT binding to striatal dopamine transporters in rat brain: a validation study. NuCl Med Biol 2006; 33: 409-11.

37 Chang L, Ernst T, Strickland T, Mehringer CM. Gender effects on persistent cerebral metabolite changes in the frontal lobes of abstinent cocaine users. Am J Psychiatry 1999; 156: 716-22.

38 Iversen L. Cannabis and the brain. Brain 2003; 126: 1252-70.

39 Tunving K, Thulin SO, Risberg J, Warkentin S. Regional cerebral blood flow in long-term heavy cannabis use. Psychiatry Res 1986; 17: 15-21.

40 Solowij N. Cannabis and Cognitive Functioning. Cambridge University Press, 1998.

41 Scholey $A B$, Parrott $A C$, Buchanan $T$, Heffernan TM, Ling J, Rodgers J. Increased intensity of Ecstasy and polydrug usage in the more experienced recreational Ecstasy/MDMA users: a WWW study. Addict Behav 2004; 29: 743-52.

42 Rothman KJ. No adjustments are needed for multiple comparisons. Epidemiology 1990; 1: 43-6. 